\title{
Modeling and CFD Analysis of Air Flow through Automotive Turbocharger Compressor: Analytical Approach and Validation
}

\author{
M.I. Soliman*, A.A. Emara ${ }^{\dagger}$, E.M. Abdel Razek ${ }^{\ddagger}$, H.A. Moneib ${ }^{\S}$
}

\begin{abstract}
In this study an effort was made to develop a flow simulation modeling and performance prediction for a centrifugal compressor stage of a heavy-duty D. I. diesel engine. The model is implemented in simulation software in MATLAB language. Additionally, a contribution of this paper to demonstrate that off-design performance of a centrifugal compressor stage in a turbocharger system can be accurately simulated using commercial CFD software, with design software, FLOEFD to generate high quality meshes and model solving. The vector plots, contour plots and stream line plots are generated for better understanding of fluid flow through centrifugal compressor stage. Correlation coefficients have been introduced in the calculation program, in order to be closer to the CFD simulation results. The results obtained from mathematical computation model were validated with the CFD analysis and experimental results performed using a test bench for the variation of the performance parameters such as isentropic efficiency, power input, and total pressure ratio with mass flow rate, the results are also presented in graphical form. The results reveal that reasonable agreement between mathematical models, the numerical results obtained from the CFD simulations and the real measurements; the maximum difference never exceeds $5 \%$. The results indicate that the developed mathematical computation model can yield better predictions of performance for a centrifugal compressor stage in a turbocharger system.
\end{abstract}

Keywords: centrifugal compressor stage, computational fluid dynamics (CFD), heavy duty diesel engine, experimental work, mathematical computation model.

\section{Introduction}

In the last decades, charging the engine is an almost diffused strategy towards the performance rise. This may be archived with a compressor before the intake air box increasing the air (or mixture) pressure and introducing a higher mass in the cylinders; this is a great advantage, improving engine power, reducing fuel consumption and decreasing emissions. However, because of complex geometry of the turbocharger, it cost a lot of time and labor power to adopt experiment to gain relevant data of performance. So, it becomes more economical to simulate turbocharger internal flow field and analysis data. Mathematical modeling and Computational Fluid Dynamics (CFD) is a cost affected tool to provide detailed flow information inside the

\footnotetext{
Research Eng., Mechanical Power Engineering Department, Faculty of Engineering, Mataria, Helwan University, Cairo, Egypt; Mostafasoliman780@ gmail.com

$\dagger$ Dr., Mechanical Power Engineering Department, Faculty of Engineering, Mataria, Helwan University, Cairo, Egypt; Aaarazek@gmail.com

* Assoc. Prof., Mechanical Power Engineering Department, Faculty of Engineering, Misr University for Science \& Technology, Cairo, Egypt; sayedm2000eg@yahoo.com

$\S$ Prof., Mechanical Power Engineering Department, Faculty of Engineering, Mataria, Helwan University, Cairo, Egypt; Hany.moneib@gmail.com
} 
complete turbocharger. Through looking up the literature of different researches, the turbocharger compressor geometry has been the subject of numerous mathematical and numerical studies, the reason for this: is that it's strongly affects the overall performance, stability, operating range and the location of the best efficiency point of the compressor. Prasad, V. [1] and Bhardwaj, S. [2] studied the compressor flow characteristics numerically and experimentally showing the difference between the experimental and numerical results which were less than 2\%, on the other hand, Demidov, V and kolchin, A. [3] discussed the compressor turbocharger mathematical molding illustrating the basic geometrical dimensions for the volute and air flow characteristics throughout the compressor passages. However, both of Prasad, V and Demidov, $\mathrm{V}$ and kolchin, A did not discuss the effect of volute cross section geometry on the air flow, the thing that was covered by Abdelmadjid, C [4] Showing that higher peak efficiency, obtained in the case of tangential inlet and wider operating range is observed in the case of modified circular case. The operating range is limited between the surge and choke boundaries, Harley, P X L. Spence, S W T and Early, J [5] and Bergqvist, S [6] simulated the surge and choke limits using CFD techniques, both of those limits can be defined using the compressor map. In the present work, a developed mathematical computation modeling for flow simulation and performance prediction through turbocharger (compressor) stage of a six cylinders heavy duty D.I. diesel engine. The investigated turbocharger compressor stage was composed of casing, diffuser, and impeller. Mathematical computation model were validated with numerical results of the simulation obtained from CFD analysis and experimental results.

\section{Methodology}

This study consists of three main parts and they are done in a specific order. The first part has the purpose to develop a mathematical computation model for the flow behavior and performance prediction from inlet to the exit of a centrifugal compressor stage consisting of all the components in place. The second part, involves an investigation of numerical flow simulation and performance characteristics through turbocharger (compressor) stage using FLOEFD flow simulation as computational fluid dynamics (CFD) software. Simulation of the off-design performance of the centrifugal compressor stage was considered for six off- speeds (rpm):60\%,70\%, 80\%, 90\%, 100\% and $110 \%$ of design speed and the various performance parameters concerning: pressure, temperature distribution and velocity profiles on the blades, isentropic efficiencies, Power input and pressure ratio are presented. In addition, correlation coefficients have been introduced in the mathematical calculation program, in order to be closer to the numerical simulation. A discussion for the second part will be done before continuing to the third part. In the third and final part mathematical computation model were validated with numerical results of the simulation obtained from CFD analysis and the experimental results performed using a test bench. Furthermore, discussion and conclusions can be found.

\section{Governing Equations of the Developed Mathematical Simulation Model}

The maximum power a diesel engine can deliver is limited by the amount of fuel that can be burned efficiently, thus increasing fresh air mass flow rate leads to more engine power, so the compressor is primarily used to boost inlet air. This study deals with mathematical simulation modeling and performance prediction of a turbocharger compressor stage for a six cylinders heavy duty D. I. It is modeled in a semi empirical way and consists of differential algebraic equations (1-4) based on the literature [4, 7 and 3] The pressure ratio and adiabatic efficiency are calculated on the basis of the total static pressures $\mathrm{P}_{\text {total }}(\mathrm{Pa})$ and total (stagnation) temperatures $\mathrm{T}_{\text {total }}(\mathrm{K})$ at both the inlet and the outlet of the compressor. 


$$
\begin{gathered}
\pi_{C}=p_{5} / p_{a_{i n}} \\
\eta_{a d . C}=T_{0}\left(\pi_{c}^{\frac{K-1}{k}}-1\right) /\left(T_{5}-T_{0}\right) \\
P_{5}=P_{4}\left(1-\frac{L_{r, s c}}{R_{a} T_{5}} \cdot \frac{k-1}{k}\right)^{k /(k-1)} \\
T_{4}=T_{2}\left(P_{4} / P_{2}\right)^{\left(n_{d}-1\right) / n_{d}}
\end{gathered}
$$

As shown from the equations (5 -8), Where $\mathrm{Ga}(\mathrm{kg} / \mathrm{s})$ and $G a_{\text {corrected }}(\mathrm{kg} / \mathrm{s})$ are the discharge air mass flow rate and corrected discharge air mass flow rates respectively at the impeller inlet. $T_{1}$ Total, inlet $(\mathrm{K})$ is the total (stagnation) temperature at the impeller inlet, and $P_{a}$ in total, inlet $(\mathrm{Pa})$ is the total (stagnation) pressure at the impeller inlet. The model described in this section is implemented in simulation software in MATLAB language to predict the turbocharger compressor stage performances. The developed simple model offers a relatively accurate performance prediction. However not accurate enough compared to experimental results, thus all performance characteristic magnitudes will be mathematically calculated and presented as a function of mass flow rate and turbo speed in order to be correlated based on the CFD and mathematical results.

$$
\begin{gathered}
\mathrm{Ga}=\frac{\alpha \varphi_{s} l_{0} N_{e} g_{e}}{36 \times 10^{5}} \\
G a_{\text {corrected }}=G a+\delta \\
T_{1}=T_{a_{\text {in }}}+\frac{v_{\text {ain }}^{1}-v_{1}^{2}}{2 c p} \\
P_{a_{\text {in }}}=P_{0}-\Delta P_{\text {in }}
\end{gathered}
$$

\section{Computational Fluid Dynamics (CFD) Analysis}

Computational fluid dynamics (CFD) allows performing detailed calculations of any system in which fluids are involved. The equations governing gas dynamics are the expressions of energy conservation and thermodynamics laws. Simulation is based on the turbulence model of the non-stationary 3D flow. Gases are defined as being compressible viscous fluids. The standard model $\mathrm{k}-\varepsilon$ is used to solve the flow problems reigning inside the turbocharger compressor stage. Equations governing the inflow model, including the mass conservation equation, momentum conservation, and the energy conservation equation, are summarized in conservative form of the Naviere Stokes equations [10][11].

\subsection{Mesh Generation}

In this study, commercial CFD tool FLOEFD is applied. FLOEFD has the advantages of importing geometry directly from a computer associated design (CAD) program such as SOLIDWORKS. First, 3D geometry of the compressor stage is built using the Solid Works (SW) software as shown in Fig.1. The models created by SW are imported in (FLOEFD) to build the grid for the final calculation of simulation; the high-quality hexahedral mesh is selected for the meshing of compressor as shown in Fig.2, Taking into consideration that the mesh size must be kept small in order to complete the simulations in a reasonable time. However, a coarser mesh could negatively affect the accuracy of the results. Therefore, a mesh density study based on literature survey [12] [14] was conducted to gauge the amount of mesh dependent error for mesh sizes in the range of 300000 to 500000 cells with one hundred cell step in each solving run until the resulting difference between the mesh sizes investigated was in general no more than $2 \%$ for the variables of interest. Therefore, the mesh used in the simulation contained a total of cells 500000 , cell volume is $2.779 \times 10^{-8} \mathrm{~m}^{3}, 289245$ nodes, 1586290 elements. 


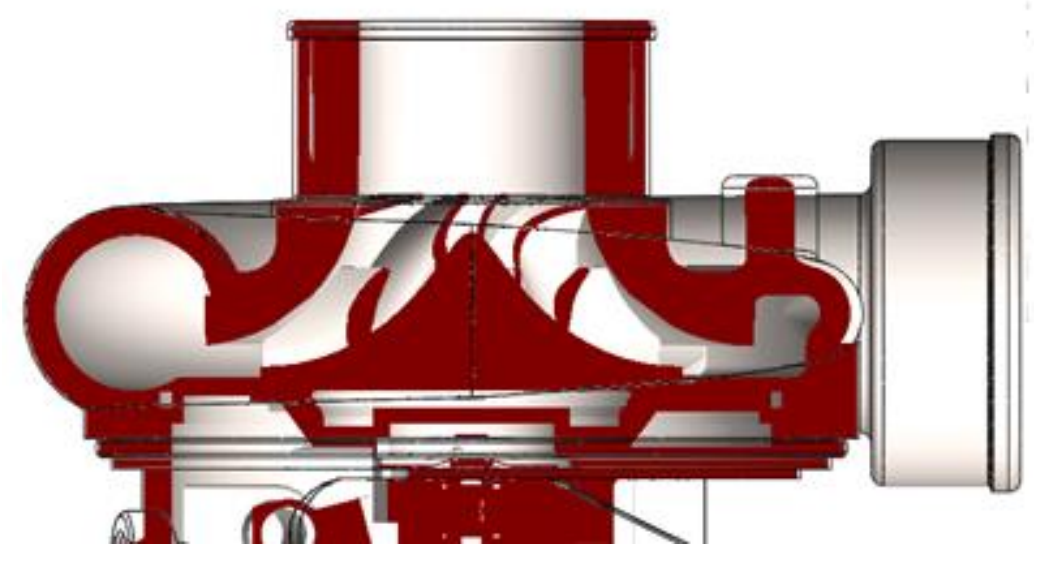

Fig. 1 The centrifugal compressor geometrical model

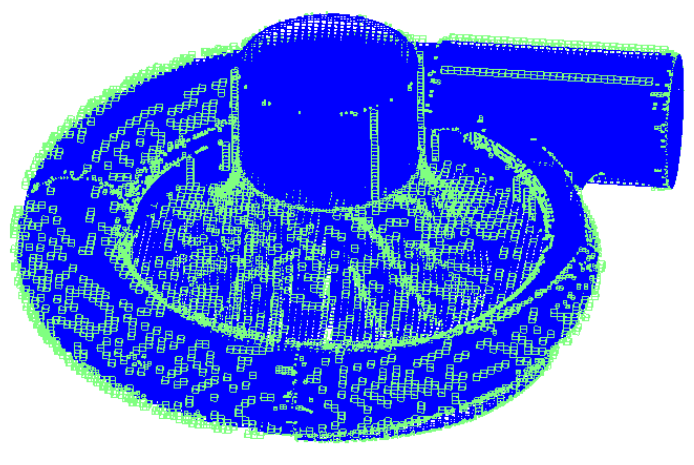

Fig.2 Fluid cells (Blue) and partial cells (green) meshing

\subsection{Boundary Conditions}

The compressor is intended to work for atmospheric conditions and should deliver a total to static pressure ratio of 1.76. As a result, the atmospheric pressure is imposed as total inlet pressure, and the total inlet temperature is fixed at $298 \mathrm{~K}$. The inlet flow is axially oriented. At the outlet a static pressure is imposed corresponding to the required pressure ratio. The boundary was defined as a subsonic, standard k- $\varepsilon$ turbulence model with turbulence intensity of 5\% was considered. The turbocharger compressor blades, hub and shroud were assumed as a smooth surface with adiabatic flow and appropriate rotational velocity. At the outlet a static pressure is imposed corresponding to the engine rpm and engine geometry, Compressor rpm is imposed corresponding to the engine load.

\subsection{CFD Results and Discussion}

Simulation of the centrifugal compressor stage performance was considered for six off-design speeds (rpm): $60 \%$ 70\%, 80\%, 90\%, 100\% and 110\% of on-design speed and the various performance parameters concerning: pressure, temperature distribution and velocity profiles on the blades, isentropic efficiencies, Power. Compressor characteristics at $100 \%$ of the load CFD results will be illustrated in the following section and discussed. 


\subsubsection{Velocity Simulation}

Figure 4 shows the simulated air velocity streamlines, the flow is stable before it enters the compressor, when it has entered the compressor and is compressed close to the wheel the speed of the fluid becomes very high. The velocity at the impeller inlet and exit was 128 and $300 \mathrm{~m} / \mathrm{s}$ respectively. The velocity of the flow downstream the compressor is slowed down in the volute and due to the volute geometry a swirl motion is generated.
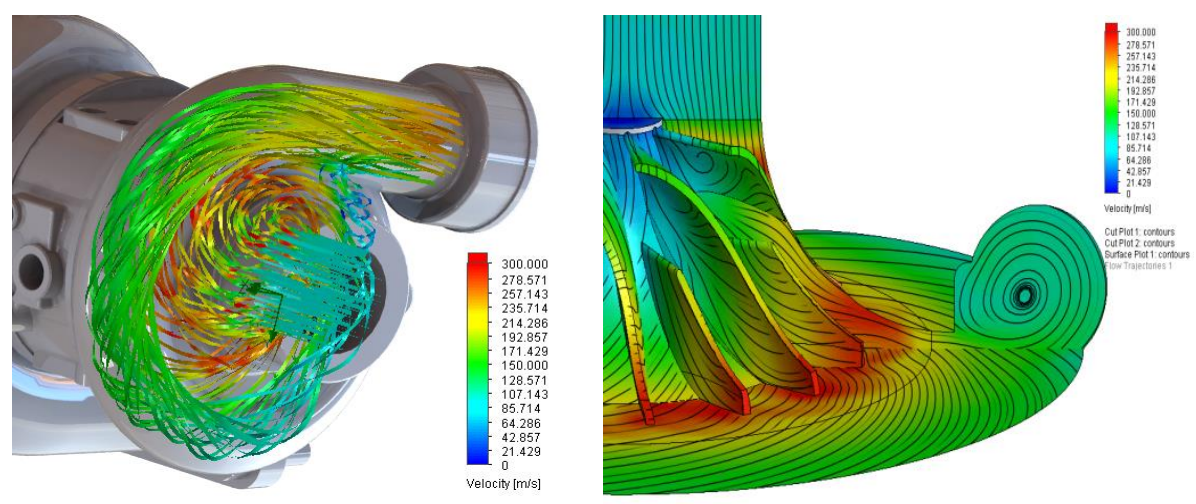

Fig.4 Air velocity simulation

\subsubsection{Temperature Simulation}

The temperature distribution before, through and after the compressor can be seen in Figure 5 for a rotation speed of $39100 \mathrm{rpm}$ with the pressure ratio 1.82. The temperature at the impeller inlet was $277 \mathrm{~K}$, at the compressor exit was $341 \mathrm{~K}$. The air is compressed which gives an increase in the air temperature in the volute results in a $362 \mathrm{~K}$
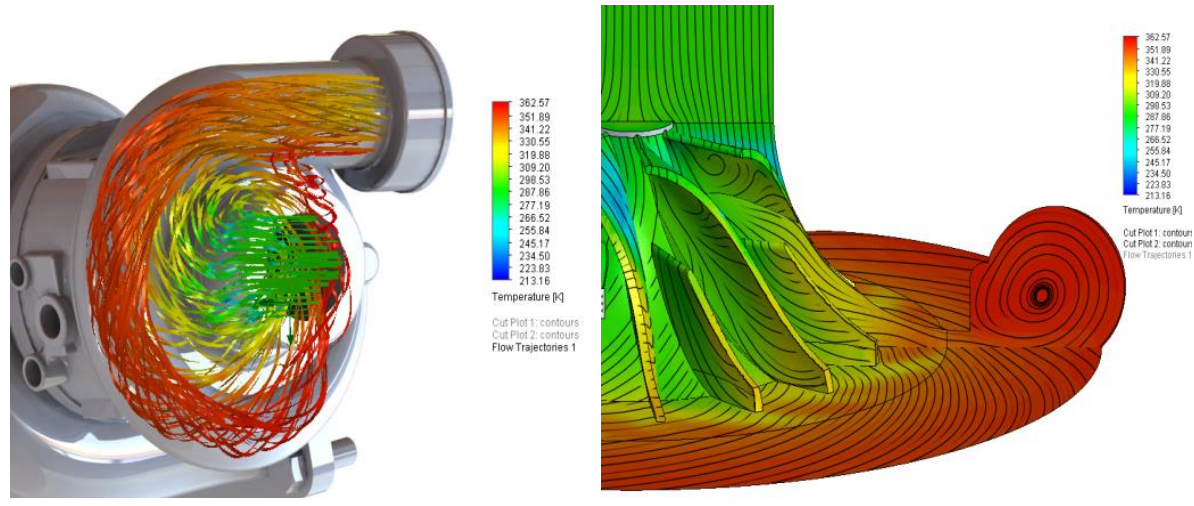

Fig.5 Air temperature simulation

\subsubsection{Pressure Simulation}

A contour plot of the pressure distribution at assembly of the compressor wheel and housing can be seen in Figure 6. The lowest pressure is found upstream-suction opining- the compressor wheel and the highest downstream the compressor wheel -volute discharge-. The distribution looks quite symmetric. The air pressure at the impeller inlet was $0.075 \mathrm{MPa}$ and $0.12 \mathrm{MPa}$ at the impeller exit. Converting the rotational dynamic energy into pressure ate the volute generating a compressed air at $0.1367 \mathrm{MPa}$. 

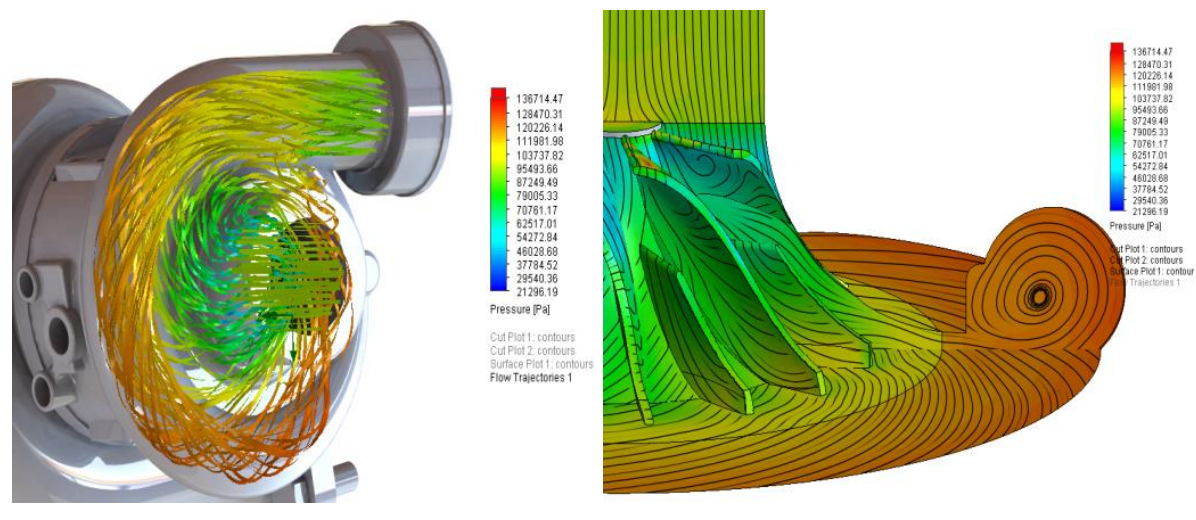

Fig. 6 Air pressure contour

\subsubsection{Density Simulation}
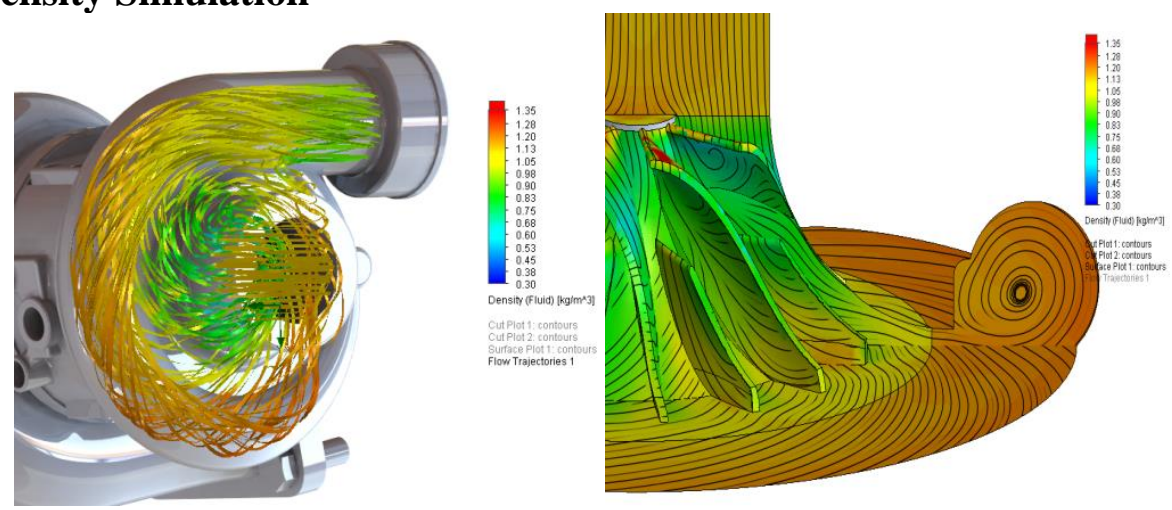

Fig.7 Air density contours

Density increases in thedownstream the compressor due to the increase of the pressure in other words as shown in Figure.7. It happens this way due to the conversion of the kinetic energy into a static energy which leads to an increase in both of the pressure and density causing maximum air density $1.35 \mathrm{~kg} / \mathrm{m}^{3}$ downstream the compressor wheel However, at the volute exit the density intend to decrease due to the effect of engine suction in the air intake manifold.

\section{Numerical and Mathematical Results Comparison}

In stage two of the study a comparison between the numerical, Mathematical calculations showing the differences between both, providing a closer vision of the engine power prediction. Mathematical model (1) can give a very good prediction for the compressor behavior, However there always a differences between the realty and what mathematics says, the reason why this is happing is that the mathematical models neglect the effect of some parameters like circulation, volute area cross section, blade airfoil cross section effect, intake manifold pressure, initial state and more, that's why a CFD model is a must aiming to having a completer study without a need for experimental expensive methods. Comparison between both of mathematical and numerical results will be discussed showing the differences between them, giving us a clear vision for the neglected parameters effect. 


\subsection{Pressure Comparison}

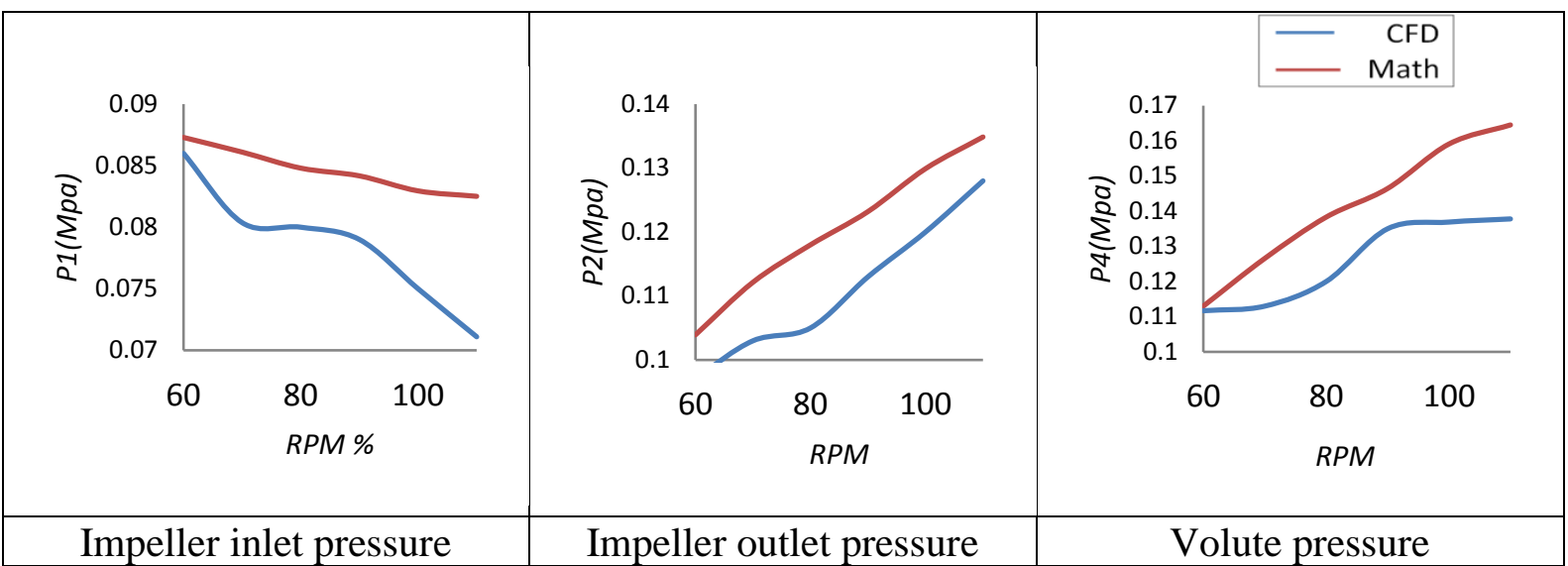

Fig 8. Impeller inlet, impeller outlet and volute pressures

The flow static pressure across the compressor is lower than calculated mathematically; at $100 \%$ of load the pressure at the impeller inlet is lower by $10 \%$ of the calculated due to compressor suction effect and high-speed entry flow, at the impeller outlet is lower by $8 \%$ and at the volute pressure is $16 \%$ lower than the mathematical calculated because of the engine suction pressure in the intake manifold.

\subsection{Velocity Comparison}

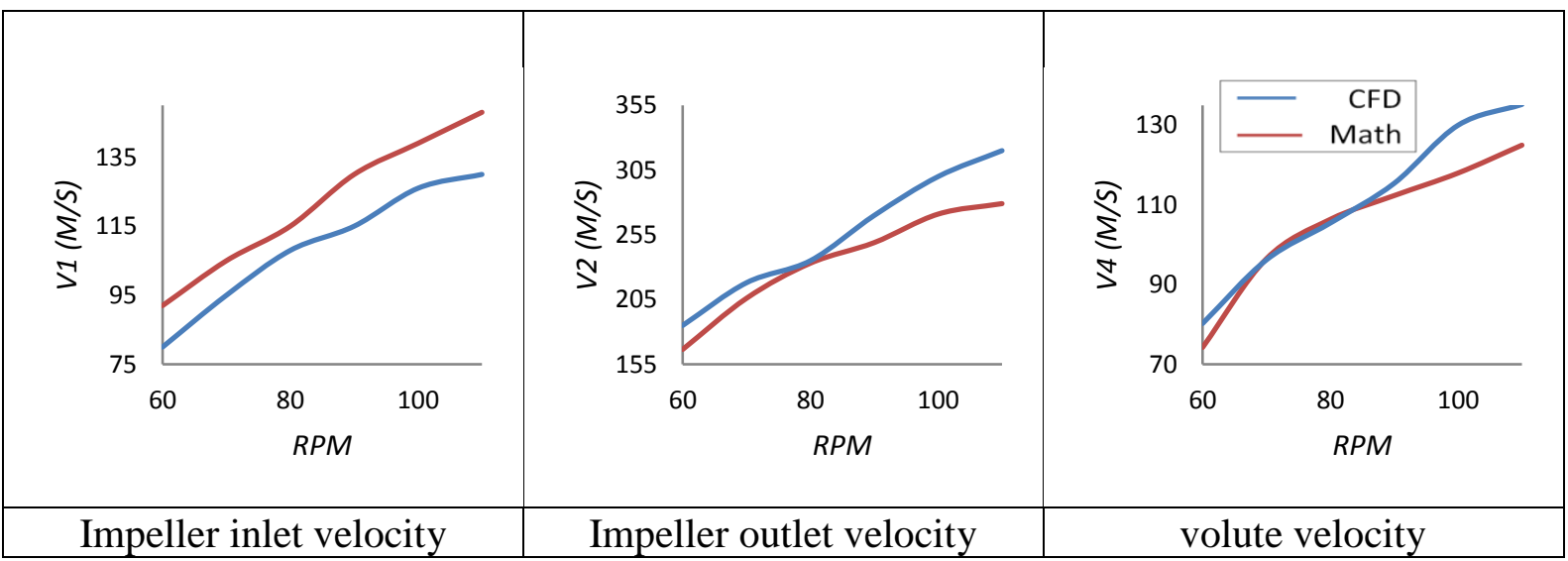

Fig 9. Impeller inlet, impeller outlet and volute velocities

Due to the boundary conditions and the pressure reduction the flow velocity across the compressor is higher than the calculated, following the first law of thermodynamics the velocity head increased with the reduction of the pressure, at the impeller inlet the flow is higher with $9 \%, 9.6 \%$ at the impeller outlet, and $9 \%$ at the volute higher velocity than the calculated. 


\subsection{Density Comparison}

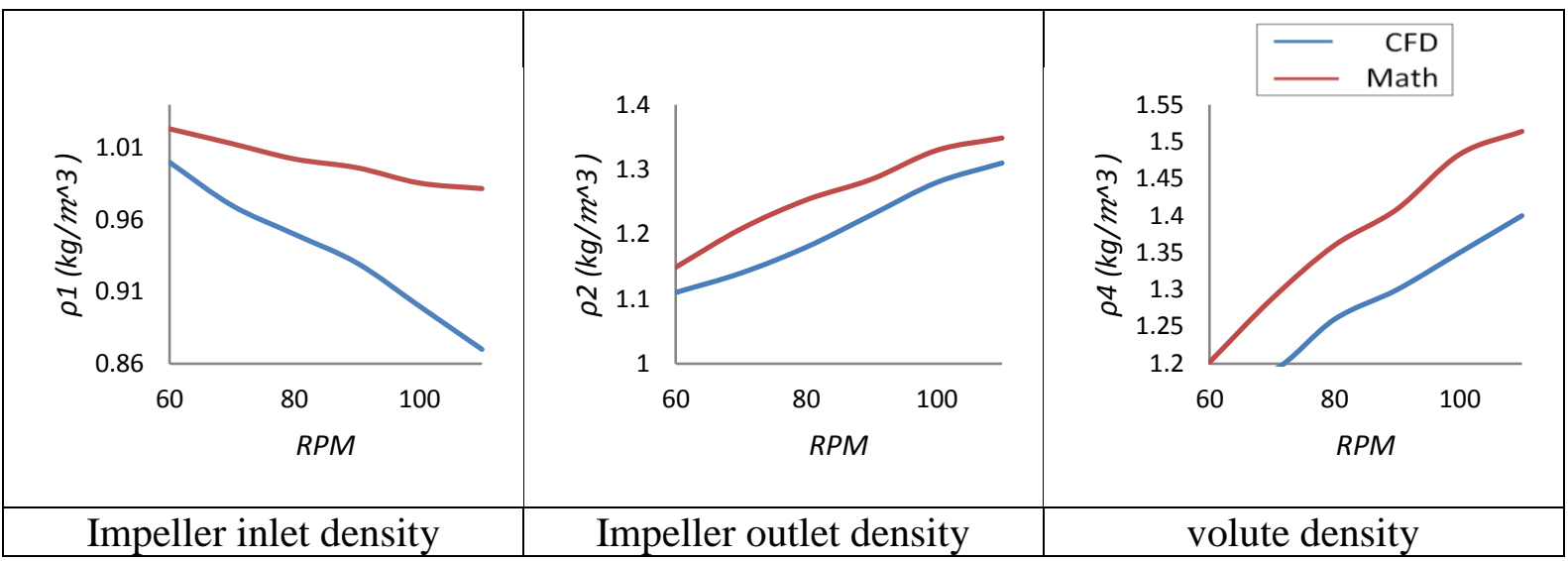

Fig 10 Impeller inlet, impeller outlet and volute densities

As a result of static head reduction and velocity, the density across the compressor flow passage is less than the calculated mathematically, Making 9.5\% difference at the impeller inlet, $3.8 \%$ difference at the impeller outlet and $9.8 \%$ difference at the volute discharge.

\subsection{Temperature Comparison}

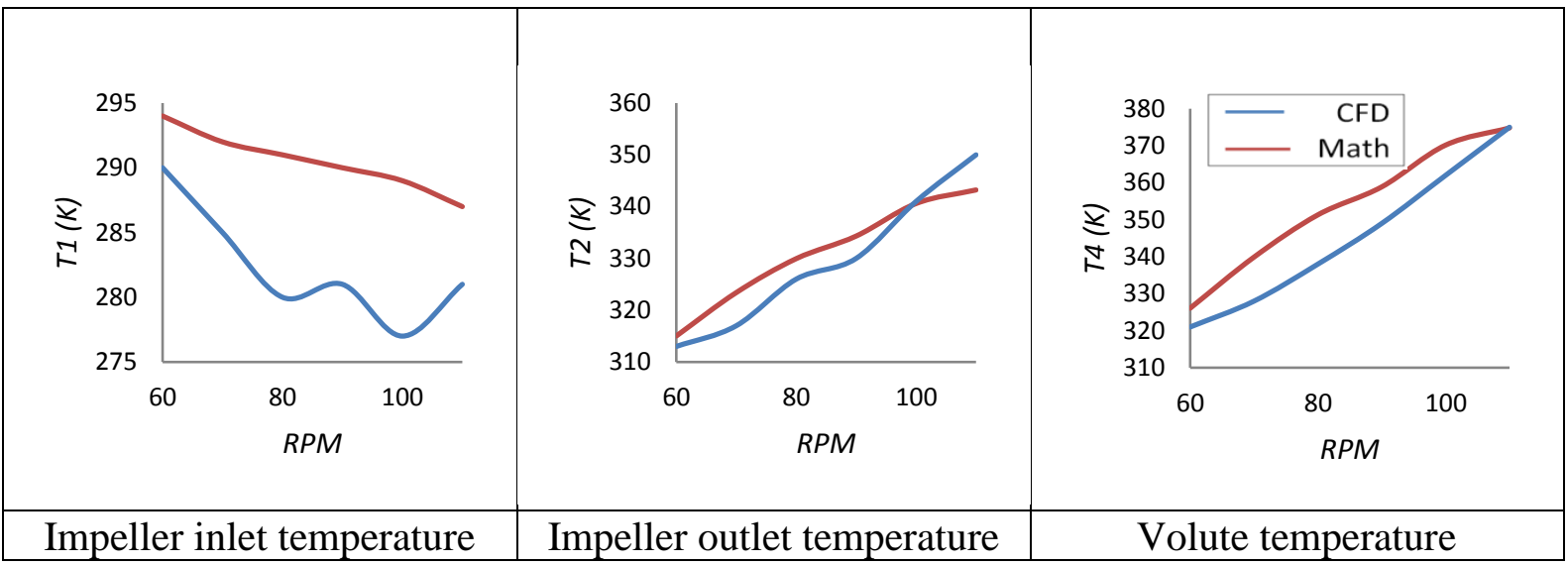

Fig 11 Impeller inlet, impeller outlet and volute temperatures

CFD temperature results are less than the mathematical predictions as an effect of static pressure reduction across the whole system, at impeller inlet a $4 \%$ difference, at impeller outlet less than $1 \%$, and $2.5 \%$ difference between numerical and mathematical calculations.

\subsection{Compressor Performance Comparison}

Compressor Performance Comparison that includes isentropic Efficiency, Consumed Power, Pressure difference and mass flow rate shows a noticeable error between Mathematical and Numerical analysis, Nakhjiri et al. [13] faced similar issue and also got similar error values despite using mathematical correction factors. 


\subsubsection{Mass flow rate}

Despite the air density decrease in, the air velocity increase led to an increase in the delivered mass flow rate by $14.3 \%$.

\subsubsection{Compressor power}

Due to the increase in the mass flow delivered the power consumed by the compressor is higher than the calculated mathematically by $34 \%$ at full load.

\subsubsection{Pressure difference}

Although both inlet pressure and outlet pressures are changed but still the ratio between the pressures differences numerical and Mathematical is $35 \%$ at full load.

\subsubsection{Compressor Efficiency}

Based on equation (2) and comparing between numerical and mathematical results shows that $20 \%$ lower efficiency at full load due to the errors in pressure difference and Temperature

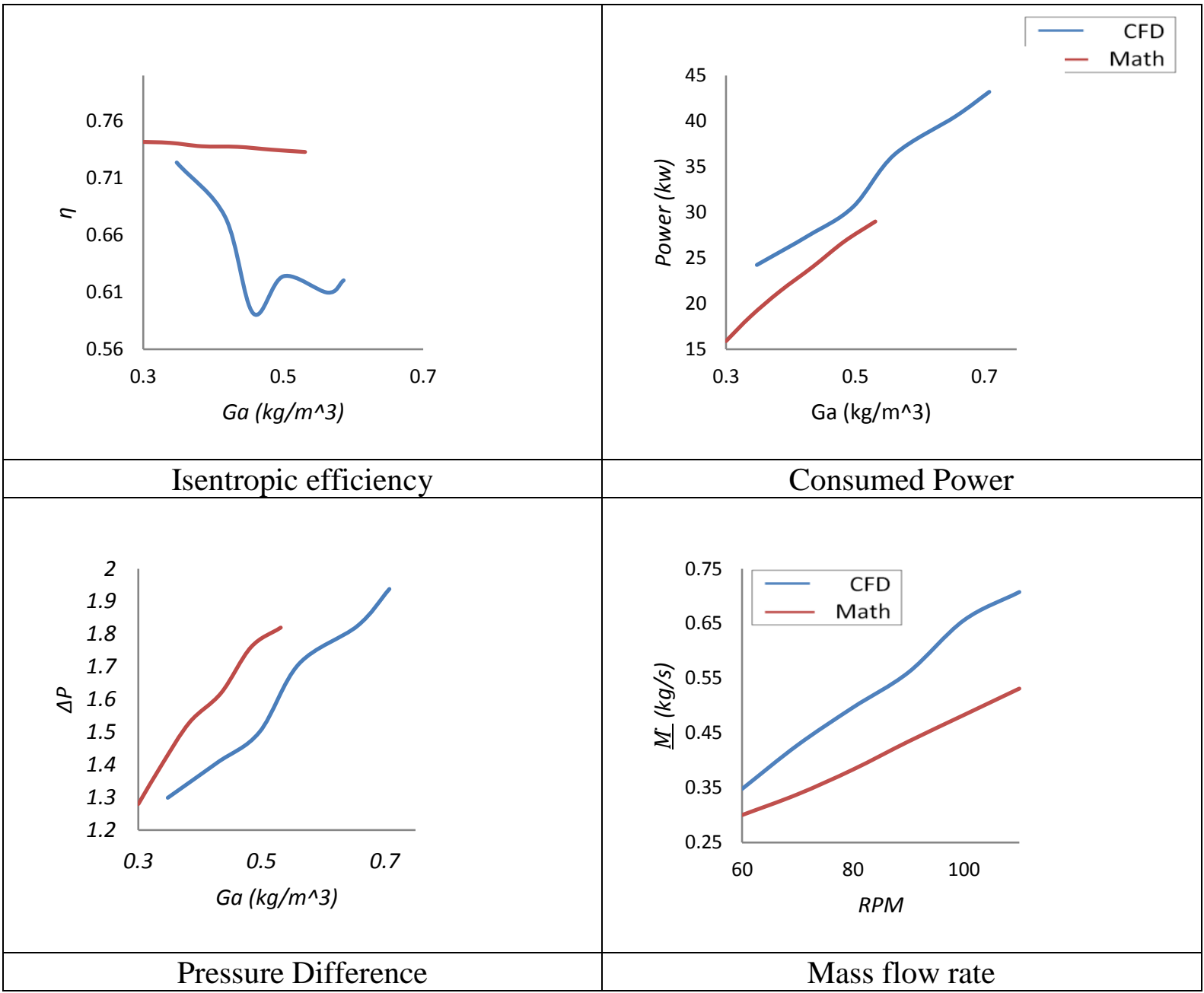

Fig 12 Performance comparison

\section{Discussion and Correlation Coefficients}

The aim of charging engines is to introduce more air mass flow rate into the combustion chamber consequently more fuel, aiming to maintain the same air to fuel ratio (14.7), in other words introducing more air to the engine with fixed value of fuel mass flow rate will decrease the fuel to air ratio value leading to less break power as based on equation (9).

Back to the result summary of mathematical and numerical comparison there is a difference between the mathematical and numerical models mainly because of neglecting the intake and discharge pressures. 
Since $\quad B P=\eta_{b t h} \times \eta_{V o l} \times \eta_{M} \times \rho_{b . c} \times \frac{F}{A} \times C V_{F} \times V_{s t} \times \frac{N}{60 n} \times i$

Thus, by neglecting the changes in the efficiencies we may simplify the pervious equation to compare engine break power expectations as a function of air density before the cylinder and Fuel to air ratio

$$
\frac{B P_{N}}{B P_{M}}=\frac{\rho_{b . c_{N}}}{\rho_{b . c_{M}}} \times \frac{\frac{F}{\bar{A}_{N}}}{\frac{\bar{A}_{M}}{A_{M}}}
$$

So, if the desired output break power is to be $360 \mathrm{hp}$ it shall be $241 \mathrm{hp}$ instead.

\subsection{Correlation Coefficients}

In order to close the error between numerical and mathematical results coefficients shall be introduced in the mathematical model to achieve more durable Mathematical model and save time of CFD studies.

\subsubsection{Inlet pressure}

In the calculation the inlet speed is higher than the calculated the thing that led to lower static pressure In order compensate this difference in the results a correction factor $b=0.9278$ must be calculated by equating $P_{1}$ polynomial equation and $P_{1 c}$

$$
\begin{aligned}
\mathrm{P}_{1} & =\mathrm{P}_{a_{i n}}\left(\mathrm{~T}_{1} / \mathrm{T}_{a_{i n}}\right)^{n_{\text {in }} /\left(n_{\text {in }}-1\right)} \\
P_{1 c} & =6 P_{1}
\end{aligned}
$$

Correcting the inlet pressure will in turn change the inlet density.

\subsubsection{Volute exit pressure}

Since the pressure can be defend as a resistance to flow the discharge pressure -Manifold pressure- should has been taken into consideration in Demidov, V and kolchin compressor model. Due to the pistons movement the discharge pressure is lower than the expected thus a correction factor $\vartheta=0.8935$

$$
\begin{aligned}
P_{4} & =P_{a, i n} \pi_{c} \\
P_{4 c} & =\vartheta P_{4}
\end{aligned}
$$

Obtaining a correct value of the discharge pressure will in turn correct the values of the density and temperature

\subsubsection{Mass flow rate}

For a diesel engine $27 \%$ more air will lead to a leaner combustion engine in other words the engine will perform with less power than the expected unless more fuel is presented to the engine to retrace the determined fuel to air ratio.

Final correction factor $\delta$ shall be added to the calculated mass flow rate, $\delta$ is a function of the inlet pressure and discharge pressure thus plotting two (Numerical and Mathematical) polynomial equations we shall obtain the correct value of $G_{a c}=G_{a}+\delta$

where

is the discharge mass flow rate.

$$
G_{a}=\frac{\alpha \varphi_{\mathrm{s}} \mathrm{l}_{0} \mathrm{~N}_{\mathrm{e}} \mathrm{e}_{\mathrm{e}}}{36 \times 10^{5}}
$$


Figures 13 and 14 illustrate $G_{a} \& G_{a c}$ in a function of inlet and discharge pressures

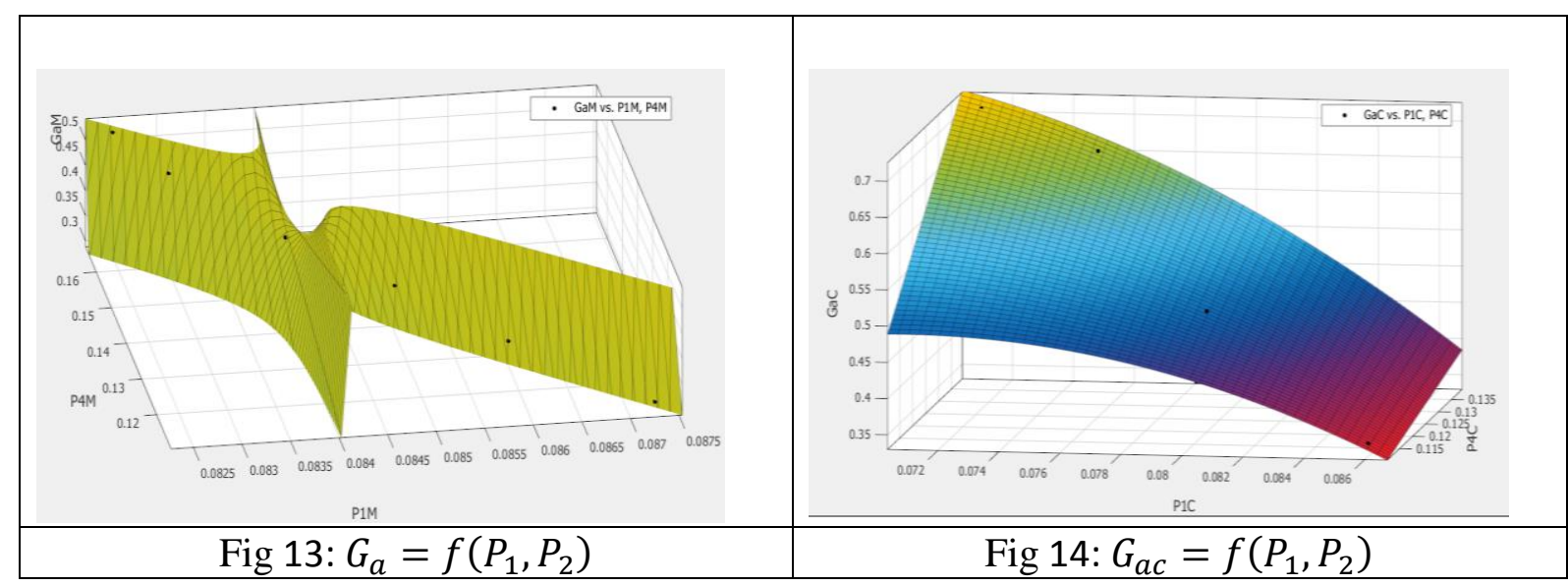

Knowing these two equations we can obtain

$$
\boldsymbol{\delta}=706053.4 P_{1}^{2}+65378.7 P_{1} P_{4}-5495.15 P_{4}-128364.9 P_{1}+5801.269
$$

Using these correction factors within the Demidov, $\mathrm{V}$ and kolchin. A mathematical model we will obtain correct values of mass flow rate $-3 \%$ error - and pressures, temperatures, densities, and in turn correct values of efficiency, power required.

\section{Experimental Setup}

The turbocharger chosen to perform this study with the reference Holset HX 80 was fitted on a heavy-duty D. I. diesel engine; (360 hp@2000 rpm and 1300 Nm@ 1450 rpm), featuring a mechanical injection system, more details about the main features of the engine and the turbocharger are listed in Table 1. The engine was instrumented on a high-dynamic test bed which was installed at the Faculty of Engineering R\&D Center in Helwan University, Cairo, Egypt. The engine was fully equipped for measurements of all standard operating parameters (torque, speed, air mass flow), but also of the static pressure and temperature in the most relevant locations along the intake and exhaust system, as well as of high frequency pressure data at the same locations and inside each cylinder. Additionally, Cooled pressure transducers were used in order to obtain an accurate measurement of the gas pressure conditions at the compressor inlet and outlet. In addition, the compressor rotating speed was also recorded in order to complete the information related to the turbocharger. Tests were carried out at different engine speeds and loads corresponding to the whole operating range of the engine. In every performed test, pressures were recorded in different points and the corresponding air mass flow and fuel flow were measured. The compressor discharge and turbine inlet headers were fitted with insulation to minimize heat loss to the surroundings, and maintain accuracy of the hightemperature measurements. A sketch of the engine layout and locations of the transducers used during the measurement campaign is shown in Fig.15. All instruments are connected to a Data Acquisition Card (DAQ) to continually monitor the turbocharger under test. In addition to monitoring temperatures and pressures of the turbine and compressor conditions, the DAQ monitors bearing and jacket water conditions for stability and safety. The complete performance profile of a turbocharger is determined from temperature and pressure instruments, and the related thermodynamic and fluid mechanic properties. A DAQ is programmed to compute flow and performance calculations of the operating turbocharger and record the performance data 
for test reporting, in accordance with the Test Standard for Testing Large-Bore Engine Turbochargers Chapman et al. 2005[12]. For every test condition, the air mass flow, fuel mass flow, and compressor inlet temperature and compressor outlet pressure were measured by the sensors of the installation. As the different tests were performed under steady state operations, the power consumed by the compressor is equal to the power developed by the turbine including turbocharger mechanical losses. So, the adapted power was obtained for the compressor power as:

$$
P_{\text {comp }}=\left[\frac{k}{k-1} R_{a} T_{a_{i n}} Q_{E X P}\left(\pi_{c}^{(k-1) / k}-1\right)\right] / 1000 \eta_{\text {ad.c }}
$$

Small differences can be observed between measured and interpolated data. On the other hand, inherent sensor uncertainties exist in both type of installation. As the parameters calculated to describe the compressor performance are combinations of various measurements, results can be slightly influenced. On the other hand, some heat transfer effects [10], especially in the low speed and low load region, and differences between compressor behavior under steady or under pulsating flow conditions [12], affect the compressor efficiency measurement.

Table 1: Basic engine and turbocharger compressor characteristics

\begin{tabular}{l|l}
\hline \hline Engine type & Heavy duty \\
\hline Cooling type & water cooled \\
\hline Number of cylinders & 6 cylinders in line \\
\hline Number of stroke per cycle & 4 \\
\hline Bore [mm] x Stroke [mm] & 150 x180 \\
\hline Compression ratio & $15: 1$ \\
\hline Rated brake power hp @ rpm & 350 @ 1800 \\
\hline Fuel injection system & Mechanical-Direct injection \\
\hline Injector pressure [bar] & 210 \\
\hline Number of holes per nozzle & 10 \\
\hline Nozzle hole diameter [mm] & 0.3 \\
\hline Start of injection & $34^{\circ}$ BTDC \\
\hline Turbocharger type & HOLSET HX80-3594117 \\
\hline Number of full blades & 8 \\
\hline Number of splitter Blades & 6 \\
\hline Impeller outlet diameter & $145 \mathrm{~mm}$ \\
\hline Diffuser outlet diameter & $193 \mathrm{~mm}$ \\
\hline Impeller outlet vane height & $9 \mathrm{~mm}$ \\
\hline Inlet hub diameter & $24 \mathrm{~mm}$ \\
\hline Impeller axial length & $63 \mathrm{~mm}$ \\
\hline Inlet mean line blade angle & $45^{\circ}$ \\
\hline Outlet blade angle (back sweep) & $75^{\circ}$ \\
\hline \hline
\end{tabular}




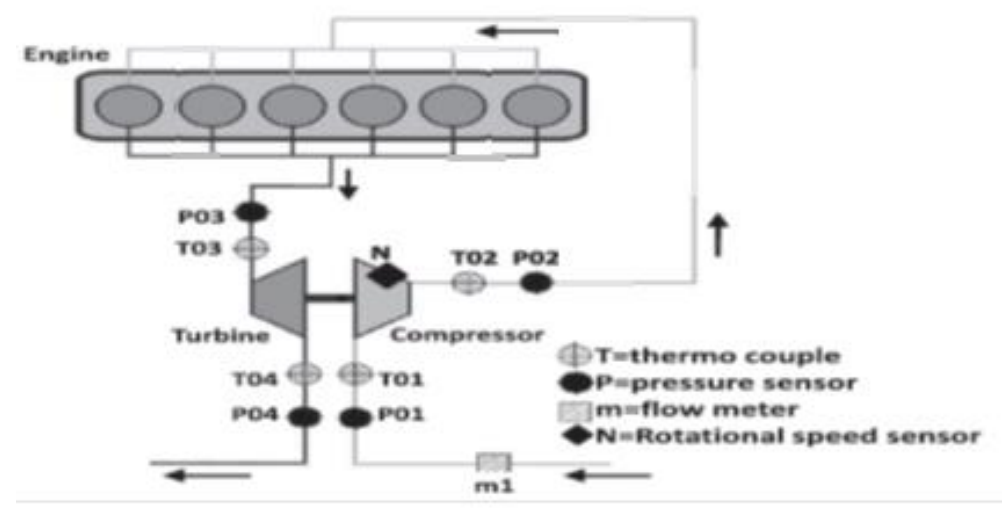

Fig 15: Test bench layout

\section{Validation of Performance Predictions with Experimental Data}

Experiments shows that the engine suffers from lower power than the expected - $246 \mathrm{hp}$ - due to un-burnt diesel fuel passing to exhaust stream -white exhaust-, gradually increasing the fuel mass flow rate into the engine, the horse power increased and the exhaust became almost colorless with $357 \mathrm{hp}$.

The results obtained from the corrected mathematical computation model, were validated with CFD analysis and the experimental results determined on a test bed for performance parameters such as polytrophic efficiency, power input, and total pressure ratio versus mas flow rate. These results will be presented in this section as shown in Figures 15 to 18 for turbocharger design speed of $39000 \mathrm{rpm}$ (Engine Full load). The first performance measure investigated was the total to static pressure ratio versus the corrected mass flow rate. This is a ratio of the static pressure ratio at the exit divided by the total pressure at the inlet respectively. As shown in Figure 15 total pressure ratio of a centrifugal compressor stage as estimated by mathematical computation code almost complies, with negligible variation of $0.3 \%, 0.9 \%$ with CFD tools and experimental results respectively. Figure16 shows mathematical computation code on the polytrophic efficiency of a centrifugal compressor predicted both CFD results and the experimental results closely with a variation of $0.3 \%$ and $1.4 \%$ respectively. Similarly Figure. 17 shows power input to a centrifugal compressor stage is predicted by mathematical computation code which compares closely with CFD and experimental results with a variation of $1.7 \%$ and 4.4. It is clear from these figures that and mathematical computation results are closely compared to the CFD numerical simulation results and the obtained experimental values and the difference doesn't exceed $5 \%$ or less for all the calculation points. This means that the flow in the compressor stage can be calculated with accuracy using both CFD and mathematical calculations methods. Once again, a good overall agreement is obtained. As justified before some uncertainties can be observed but they are always included in a narrow confidence interval. This comparison confirms that the representation defined is valid to predict the turbine performance in the whole turbocharger operating conditions. 

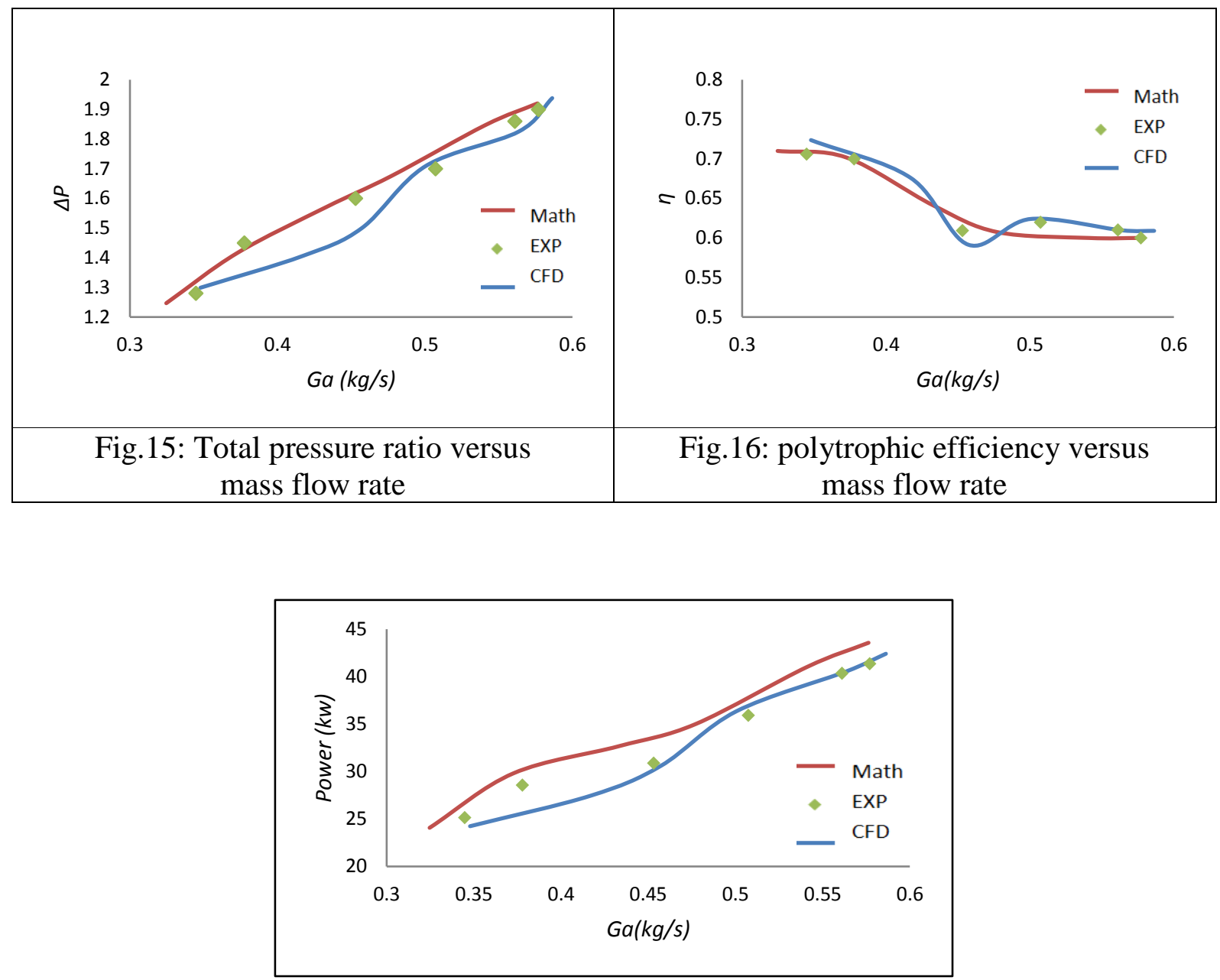

Fig.17: Power input versus mass flow rate.

\section{Conclusion}

Mathematical or physical molding for engineering designing is the easiest and fastest method of designing However, it is not accurate enough for designing and matching, on the other hand numerical molding is more accurate giving a closer vision to the designer but still it's a timeconsuming process and any small change in the model will requires more time to adopt with it. Having the accuracy of numerical analysis in a Mathematical Code will guarantee fast and easy designing and matching method for any manufacture.

This paper presents an effort to model the flow from inlet to the exit of a turbocharger compressor stage consisting of all the components in place and performance prediction by providing mathematical computation model and numerical analysis using CFD tools and these were verified by experimental work. Using of the computational fluid dynamics (CFD) gave a better understanding of the behavior of flow through turbocharger compressor stage and how it impacts the turbocharger efficiency and how the turbocharger compressor perform combined with the diesel engines.

1. A good agreement was achieved between the computational fluid dynamics (CFD), calculated and the experimental results, on the isentropic efficiency of a centrifugal compressor stage with maximum variation of $1.4 \%$. Similarly, power input to the centrifugal compressor stage predicted by CFD results which compares closely with experimental and computed results with a variation of $1.7 \%$ and $4.4 \%$ respectively. As well as total pressure ratio of a centrifugal compressor stage as estimated by CFD tools almost complies, with negligible variation of $0.3 \%$, and $0.9 \%$ with experimental and computed results respectively. 
2. An agreement between correlated mathematical model, CFD computations and the real measurements, is obtained, in addition the capability of the correlated mathematical model to predict satisfactorily the performance of a centrifugal compressor stage in a turbocharger system with maximum error $5 \%$ for most of the performance range.

3. The comparison between experimental and simulated results shows a good correlation. This result is to a large extent to be expected due to the use of adaptation coefficients, the establishment of such adaptation coefficients are however a delicate process, firstly because they must represent the real physical phenomena and secondly due to their independence. The precision obtained could be considered excessive but is quite necessary in the setting up of the complete turbocharged diesel engine simulation.

\section{References}

[1] Prasad, V.V.; Kumar, L.M. and Reddy, B.M., "Centrifugal Compressor Fluid Flow Analysis Using CFD”, Science Insights, Aug 2011.

[2] Bhardwaj, S. and Buke, Y., "Computational Fluid Dynamics Analysis of a Turbocharger System", International Journal of scientific research, May 2014, ISSN No 2277 - 8179

[3] Kolchin A., and Demidov V., "Design of Automotive Engines" MIR, Publishers, Moscow, 1984.

[4] Abdelmadjid, C. Ameur M., Boumeddane B., "CFD analysis of the volute geometry effect on the turbulent air flow through the turbocharger compressor", ELSEVIER, Energy Procedia, 36, (2013), 746 - 755,

[5] Harley, P. Spence, S. Early, J., "Inlet recirculation in automotive turbocharger centrifugal compressor,"

[6] Stephanie Berqvist, "Prediction of Turbo Compressor Maps using CFD", Chalmers University of Technology (2014)

[7] Heywood, J. B. "Internal Combustion Engine Fundamentals" McGraw-Hill, 1988.

[8] A. J. Mohamed, K. Gueorgui, and S. A. Mohamed, "Influence of intake manifold design on in-8CFD analyses and experimental investigations", Energy 36, 2701-2715, 2011.

[9] Chapman, K. S., Keshavarz, A., Shultz, J., Sengupta, J., 2005, "Test Standard for Testing Large- Bore Engine Turbochargers," Gas Research Institute, Report No. GRI-05/0026, Des Plaines, IL.

[10] S. Shaaban, J. Seume, "Analysis of turbocharger non-adiabatic performance", in: C647/027, Proceedings of 8th International Conference on Turbochargers and Turbocharging, London, 2006.

[11] Ding M.Y, Groth.C, Kacker.S and Roberts.D, "CFD Analysis of Off-design Centrifugal Compressor Operation and Performance". International ansys Conference (2006)

[12] P. Le Sausse, P. Fabrie1, D. Arnou, and F. Clunet, "CFD comparison with centrifugal compressor measurements on a wide operating range",EPJ Web Conference 45, 01059 (2013).

[13] Nakhjiri, M, Pelz, P and Matyschok, B , "Physical Modeling of Automotive Turbocharger Compressor: Analytical Approach and Validation", SAE International, 2011-012214(2011).

[14] Galindo.J, Fajardo.P , Navarro.R, Garca-Cuevas.L.M. "Characterization of a radial turbocharger turbine in pulsating flow by means of CFD and its application to engine modeling”, Applied Energy 103 (2013) 116-1 